\title{
V600E and Non-V600E BRAF Mutations in Colorectal Cancer: Clinicopathologic Correlations in a Single Institution
}

\author{
Cem SIMSEK${ }^{1}$, Sahin LACIN², Esin ECE², Suayib YALCIN ${ }^{2}$ \\ ${ }^{1}$ Hacettepe University, Faculty of Medicine, Department of Internal Medicine \\ ${ }^{2}$ Hacettepe University, Faculty of Medicine, Department of Medical Oncology, Ankara, TURKEY
}

\begin{abstract}
Genomic characterization of BRAF mutation in colorectal cancer (CRC) revolutionized it's management. Current knowledge regarding BRAF mutant CRC is based on the prevalent V600E mutation and mostly on Western population. However, CRC is known to be a complex and heterogenous disease. Thus, we aim to characterize the molecular, clinical and epidemiologic features of V600E as well as non-V600E BRAF mutated CRC in Turkish population. Demographic, histopathologic, molecular and clinical data of V600E and non-V600E BRAF mutant, metastatic and non-metastatic CRC cases were retrospectively collected from a tertiary Oncology hospital. Thirty cases of BRAF mutant colorectal carcinoma was identified. BRAF mutations were V600E (66.7\%), V600A (10.0\%), V600G (3.3\%), V600K (3.3\%), and L597V (16.7\%). BRAF V600E cases had similar characteristics with Western population: frequent in females (45.0\%), more proximal location (52.6\%), aggressive histopathologic features (LVI 50.0\%), and a worse prognosis (OS 13 vs 30 months, $p=0.068)$. Non-V600E BRAF mutant cases were diferred from V600E cases by being more frequent in males (50.0\%), located more distally (60.0\%), and carrying a better prognosis. This study demonstrates V600E mutation in CRC in Turkey is similar with Western population. In like manner, non-V600E BRAF mutation in CRC bears the potential to be a significant attribute for both prognostic and therapeutic implications as well.
\end{abstract}

Keywords: BRAF, V600E, Non-V600E, Colorectal carcinoma, Turkish

\section{INTRODUCTION}

Colorectal cancer (CRC) is the third leading cause of cancer-related death and its mortality has decreased with the improved screening methods and treatment modalities through the past decades. ${ }^{1}$ However, this longer survival has not been achieved for a fraction of cases, including BRAF mutated metastatic CRC, thus a better insight into these molecular subtypes is needed.

BRAF protein is a part of MAPK signaling, that takes part in cellular growth, differentiation, mi- gration, and proliferation. Most frequent BRAF mutation in human cancer is the substitution of 600th codon valine by glutamic acid (V600E). This mutation, occurring in the glycine-rich Ploop, which includes the activation segment, increases the independent kinase activity of BRAF protein, thus results in downstream activation. ${ }^{2-4}$ Other BRAF mutations with diverse activities were shown in various cancers, including malignant melanoma, non-small cell lung cancer, and Langerhans cell histiocytosis, with proposed clinical significance..$^{5-10}$ 
Mutations in KRAS and BRAF proteins are the commonest ones among MAPK pathway in CRC, reported in $40 \%$ and $10 \%$ of cases respectively with a mutually exclusive manner. V600E ${ }^{\mathrm{m}} \mathrm{BRAF}$ in CRC ( $\left.{ }^{\mathrm{V} 600 \mathrm{E}} \mathrm{CRC}\right)$ is diagnosed more frequently in female and elderly, more proximal, accompanied by lymphocytic infiltration, have mucinous and undifferentiated histology. More typically associated with serrated adenoma/methylation pathway, also has somatic microsatellite instability (MSI-H) and increased $\mathrm{CpG}$ island methylation (CIMP-H) phenotype. There is a great resemblance between ${ }^{\mathrm{mt}}$ BRAF CRC and Consensus Molecular Subtype 1 defined by Colorectal Cancer Consortium. ${ }^{11}$ NonV600E BRAF mutations in CRC ( ${ }^{\text {nonV600E }}$ CRC) have also been defined. They were suggested to be different from ${ }^{\mathrm{V} 600 \mathrm{E}} \mathrm{CRC}$ as they are associated with male predominance, distal location, MSI-H phenotype, a longer OS with a lesser propensity of peritoneal metastasis. Thus non ${ }^{\mathrm{V} 600 \mathrm{E}} \mathrm{CRC}$ was proposed to constitute a distinct subtype in CRC. ${ }^{9,12}$

BRAF mutation has impacts on prognosis and treatment in CRC. Microsatellite stable (MSS) ${ }^{\mathrm{V} 600 \mathrm{E}} \mathrm{CRC}$ was shown to carry a worse prognosis and has a shorter OS for both early and metastatic stage. They have poorer response to anti-EGFR and fluoro-pyrimidine based cytotoxic chemotherapy especially for stage II and III tumors, triple cytotoxic treatment have been advocated by some guidelines. ${ }^{9,13}$ Response to anti-VEGF agents was found to be similar to BRAF wild-type tumors. ${ }^{14}$ Newer therapeutic interventions such as combination molecular therapies targeting EGFR, RAF, MEK and PI3K molecules have been tested and reviewed extensively elsewhere. ${ }^{15}$

CRC is known to show geographic and racial disparities which is hypothesized to be induced by differences between genetic and environmental factors. Geographic heterogeneity of its incidence varying up to ten times between different continents is a convincing example. ${ }^{16,17}$ Likewise, in ${ }^{\mathrm{V}}{ }^{600 \mathrm{E}} \mathrm{CRC}$, data from Middle-Eastern and Asian populations have mentionable divergences regarding incidence, epidemiology and molecular characteristics from pre-mentioned features of ${ }^{\mathrm{V}}{ }^{600 \mathrm{E}} \mathrm{CRC}$ which were mostly derived from studies within Western populations. ${ }^{18-20}$ Our current knowledge regarding ${ }^{\mathrm{m}} \mathrm{BRAF}$ in $\mathrm{CRC}$ in Turkish population is limited. Studies to date demonstrated $0 \%$ to $30 \%$ incidence, however scarcity on clinical and histopathologic aspects of the cases. ${ }^{21,22}$

Although mortality of CRC has been decreased with better screening and treatment, there is a mentionable fraction of patients who do not benefit from the current therapeutic options. This demonstrates that there are significant gaps in our ability in the management of CRC. Delineating the different subtypes of CRC will be a valuable mean to address those gaps. The study aims to further characterize the molecular, clinical and epidemiologic features of V600E and nonV600E BRAF mutated $\mathrm{CRC}$ in the Turkish population regarding the key research question of whether or not non ${ }^{\mathrm{V} 600 \mathrm{E}} \mathrm{CRC}$ cases differ from ${ }^{\mathrm{V} 600 \mathrm{E}} \mathrm{CRC}$, and Turkish ${ }^{\mathrm{V}}{ }^{600 \mathrm{E}} \mathrm{CRC}$ differ from other data in literature.

\section{MATERIALS AND METHODS}

\section{Study Design}

This is a retrospective case-series. Colorectal cancer registry of a University-affiliated Oncology hospital was retrospectively evaluated between 01.01.2012 and 12.12.2015. Inclusion criteria required colorectal cancer patients to have carried a BRAF gene mutation and have evaluable records. Mutations were tested in different labs using PCR techniques. Patients' data including epidemiological, pathological, clinical, and survival were collected. Data gathered were analyzed with IBM SPSS ${ }^{\circledR} 25.0$ using Pearson, Fisher, Mann-Whitney $\mathrm{U}$ and Log-Ranks tests.

\section{RESULTS}

\section{BRAF Mutation Status}

Of all colorectal cancer patients in the registry, 30 cases were found to be BRAF mutated. Most frequent mutation was $\mathrm{V} 600 \mathrm{E}$ seen in 20 patients $(66.7 \%)$. Second and third commonest ones were L597V and V600A, seen in $5(16.7 \%)$ and 3 (10.0\%) patients respectively. They were followed by two other mutations, V600K and V600G, both were seen in one $(3.3 \%)$ patient. Consequently, $34.5 \%$ of the mutations in the population were nonV600E CRC ( ${ }^{\text {nonV600E }} C R C$ ). All cases were tested for RAS mutation and none were positive (Table 1). 
International Journal of Hematology and Oncology

Table 1. Subtypes of BRAF mutant colorectal cancer

\begin{tabular}{|ll|}
\hline BRAF Mutation & Number (\%) \\
\hline V600E & $20(66.7 \%)$ \\
L597V & $5(16.7 \%)$ \\
V600A & $3(10.0 \%)$ \\
V600G & $1(3.3 \%)$ \\
V600K & $1(3.3 \%)$ \\
\hline
\end{tabular}

\section{Patient and Tumor Characteristics}

Patients' age ranged from 19 to 79 years, with a median of 59.5, with slight male predominance $(53.5 \%)$. The proportion of males were similar between ${ }^{\mathrm{V} 600 \mathrm{E}} \mathrm{CRC}$ group and ${ }^{\mathrm{nonV}}{ }^{600 \mathrm{E}} \mathrm{CRC}$ groups (55.0\% and $50.0 \%, \mathrm{p}=0.068)$.

Primary tumor location was distal colon in over half of all CRC cases (51.7\%). Primary V600ECRC tumors were located proximally $(52.6 \%)$, in contrast ${ }^{\text {non }}{ }^{600 E} \mathrm{CRC}$ tumors were mostly distal $(60.0 .6 \%)(\mathrm{p}<0.1)$.

Study population consisted of mostly metastatic cases (73.3\%). For both ${ }^{\mathrm{V}}{ }^{600 \mathrm{E}} \mathrm{CRC}$ and ${ }^{\mathrm{nonV}}{ }^{\mathrm{6} 00 \mathrm{E}} \mathrm{CRC}$ tumors, liver was the most common region for metastasis. Non-metastatic cases' T stages at presentation were $\mathrm{T} 2$ in $33.3 \%$, and $\mathrm{T} 3$ in $66.7 \%$. Lymph node metastasis were seen in $50.0 \%$ of patients, with $16.7 \% \mathrm{~N} 1$ and $33.3 \% \mathrm{~N} 2$. In non-metastatic cases, stage 3 was the most prevalent stage at presentation.

\begin{tabular}{|c|c|c|}
\hline & V600E (n/\%) & Non-V600E (n/\%) \\
\hline Mucinous differentiation & $\mathrm{N} / \mathrm{A}$ & $4(40.0 \%)$ \\
\hline Perineural invasion & 3 (25.0\%) & $3(42.9 \%)$ \\
\hline Lymphovascular invasion & $6(50.00 \%)$ & 3 (42.9\%) \\
\hline Microsatellite instability & 2 (22.2\%) & $(0.0 \%)$ \\
\hline
\end{tabular}

\section{Pathological Characteristics}

Mucinous differentiation was reported in $40 \%$ of non${ }^{{ }^{6}{ }^{60 E}} \mathrm{CRC}$ tumors. Perineural invasion washigher in

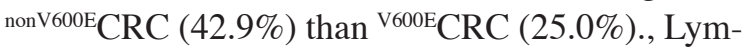
phovascular invasion was more frequent in ${ }^{6} 600 \mathrm{E}-$ CRC patients $(50.0 \%)$ than ${ }^{\mathrm{non} V 600 \mathrm{E}} \mathrm{CRC}(42.9 \%)$. Epigenetic attributes could not be comprehensively evaluated due to the retrospective nature of our study. With the available data investigating MSH2, MSH6 and MLH1 expressions, ${ }^{\mathrm{V} 600 \mathrm{E}} \mathrm{CRC}$ and non${ }^{\mathrm{V}}{ }^{600 \mathrm{E}} \mathrm{CRC}$ patients showed $10 \%$ and $22 \%$ of MSI phenotype respectively (Table 2).

Tumor markers were as CEA above $5 \mathrm{ng} / \mathrm{mL}$ in $57.9 \%$ and CA $19-9$ above $37 \mathrm{U} / \mathrm{mL}$ in $50.0 \%$ of cases. ${ }^{\mathrm{V}}{ }^{600 \mathrm{E}} \mathrm{CRC}$ and ${ }^{\mathrm{NonV}}{ }^{600 \mathrm{E}} \mathrm{CRC}$ did not differ in tumor marker expression (Table 3 ).

\section{Clinical Characteristics}

Regarding 12 non-metastatic $\left(\mathrm{CRC}^{\text {nonmet }}\right)$ patients at presentation, 6 patients' complete initial treatment regimens and radiological response data could be

\begin{tabular}{|c|c|c|c|c|}
\hline & & V600E (n/\%) & Non V600E (n/\%) & $p$ \\
\hline \multirow[t]{2}{*}{ Gender } & Male & $11(55.0 \%)$ & 5 (50.0\%) & 0.796 \\
\hline & Female & $9(45.0 \%)$ & 5 (50.0\%) & \\
\hline Age (Median) & & 62 & 58 & \\
\hline \multirow[t]{2}{*}{ Metastasis Status } & Non-metastatic & $5(25.0 \%)$ & $3(30.0 \%)$ & 0.770 \\
\hline & Metastatic & 15 (75.0\%) & 7 (70.0\%) & \\
\hline \multirow[t]{2}{*}{ CEA Levels } & $<5 \mathrm{ng} / \mathrm{mL}$ & $6(50.0 \%)$ & 2 (28.6\%) & 0.361 \\
\hline & $>5 \mathrm{ng} / \mathrm{mL}$ & 6 (50.0\%) & 5 (71.4\%) & \\
\hline \multirow[t]{2}{*}{ Ca 19-9 Levels } & $<37 \mathrm{U} / \mathrm{mL}$ & $6(46.2 \%)$ & $4(57.1 \%)$ & 0.639 \\
\hline & $>37 \mathrm{U} / \mathrm{mL}$ & 7 (53.8\%) & 3 (42.9\%) & \\
\hline \multirow[t]{2}{*}{ Tumor Location } & Distal & $9(47.4 \%)$ & $6(60.0 \%)$ & 0.518 \\
\hline & Proximal & $10(52.6 \%)$ & 4 (40.0\%) & \\
\hline
\end{tabular}




\begin{tabular}{|c|c|c|c|c|c|c|}
\hline & Gender & Age & $\begin{array}{l}\text { BRAF } \\
\text { Mutation }\end{array}$ & $\begin{array}{l}\text { Best Overall } \\
\text { Response }\end{array}$ & $\begin{array}{l}\text { Progression } \\
\text { Free Survival }\end{array}$ & $\begin{array}{l}\text { Overall } \\
\text { Survival }\end{array}$ \\
\hline FOLFOX & Female & 52 & L597V & Partial response & 18 & 47 \\
\hline \multirow[t]{2}{*}{ FOLFIRI } & Female & 74 & V600A & Partial response & 35 & 50 \\
\hline & Male & 36 & V600E & NA & NA & 13 \\
\hline \multirow[t]{2}{*}{ CAPECITABINE } & Male & 61 & V600A & Stable disease & 12 & 65 \\
\hline & Female & 60 & L597V & NA & NA & 25 \\
\hline FUFA & Male & 79 & V600E & Progression & 0 & 28 \\
\hline FOLFOX-BEVA & Female & 54 & V600E & Stable disease & 19 & 22 \\
\hline \multirow[t]{7}{*}{ FOLFIRI-BEVA } & Male & 58 & L597V & Stable disease & 13 & 33 \\
\hline & Female & 41 & V600E & Stable disease & 6 & 7 \\
\hline & Male & 62 & V600E & Progression & 6 & 12 \\
\hline & Female & 65 & V600E & Progression & 3 & 5 \\
\hline & Male & 53 & V600G & Stable disease & 13 & 24 \\
\hline & Female & 52 & V600K & Progression & 12 & 21 \\
\hline & Male & 61 & V600E & Stable disease & 6 & 37 \\
\hline \multirow[t]{2}{*}{ FOLFIRI -CETUXIMAB } & Female & 46 & V600E & Stable disease & NA & 5 \\
\hline & Male & 59 & V600E & Stable disease & 12 & 23 \\
\hline
\end{tabular}

obtained, and is as follows: Three ${ }^{\text {nonV600E }} \mathrm{CRC}^{\text {nonmet }}$ cases treated with FOLFOX (folinic acid, fluorouracil, oxaliplatin) or FOLFIRI (folinic acid, fluorouracil, irinotecan) regimens yielded a median progression-free survival (PFS) of 18 months and a median overall survival (OS) of 50 months. For two ${ }^{\mathrm{V} 600 \mathrm{E}} \mathrm{CRC} \mathrm{C}^{\text {nonmet nonV600E }} \mathrm{CRC}^{\text {nonmet }}$ cases that were treated with FOLFIRI plus antiangiogenic agent, PFS were 13 months for the former and 31 months for the following. One ${ }^{\mathrm{V} 600 \mathrm{E}} \mathrm{CRC} \mathrm{C}^{\text {nonmet }}$ patient treated with fluoro-pyrimidines alone had a shorter PFS of 3 months.

For 22 patients with metastasis (CRC $\left.{ }^{\text {met }}\right), 16$ patients medical therapy was available for review and included regimens consisting of fluoro-pyrimidines alone or with cytotoxic or anti-angiogenic agents. One ${ }^{{ }^{6000 E}} \mathrm{CRC}^{\text {met }}$ patient treated with only fluoropyrimidines had 6-month PFS and 15-month OS. Two ${ }^{\text {nonV }{ }^{600 E}}{ } \mathrm{CRC}^{\text {met }}$ patients had a OS of 25 and 65 months with single fluoro-pyrimidine. One ${ }^{\mathrm{V}} 600 \mathrm{E}-$ $\mathrm{CRC}^{\text {met }}$ patient was treated with FOLFIRI, the OS was 13 months, PFS could not be obtained. For the other two patients treated with FOLFIRI regimen, PFS were 8 and 14 months and OS were 29 and 32 months.
For thirteen patients who were administered FOLFIRI plus antiangiogenic treatment, V600ECRCmet patients' median PFS 6 months while non${ }^{\mathrm{V}}{ }^{600 E} \mathrm{CRC}^{\text {met }}$ has a 13 months with a statistically significant significance $(p=0.03)$. For the same treatment strategy, the median OS was 7 months for ${ }^{{ }^{6000 E}} \mathrm{CRC}^{\text {met }}$ and 21 months for ${ }^{\mathrm{n}}{ }^{\mathrm{V} 600 \mathrm{E}} \mathrm{CRC}^{\mathrm{met}}$ $(p=0.69)$. Best overall responses to specific regimens did not differ between ${ }^{\mathrm{V} 600 \mathrm{E}} \mathrm{CRC}^{\text {met }}$ and non${ }^{\mathrm{V}}{ }^{600 \mathrm{E}} \mathrm{CRC}^{\mathrm{met}}$ tumors for any of the various treatment strategies $(\mathrm{p}=0.76)($ Table 4$)$.

For all BRAF mutant metastatic patients (CRCmet), median OS was 21.5 months, and median PFS was 10.0 months. Regarding different BRAF mutations, ${ }^{\mathrm{V}}{ }^{600} \mathrm{CRC}^{\mathrm{met}}$ had a lower OS of 13 months compared to 30 months OS of ${ }^{\text {nonV600E }} \mathrm{CRC}^{\mathrm{met}}$, although not reaching statistical significance $(\mathrm{p}=$ 0.068). Similarly, PFS of ${ }^{{ }^{6600 E}} \mathrm{CRC}^{\text {met }}$ was also lower with 6.0 months compared to 11.5 months median PFS of ${ }^{\text {nonV600E }}{ }^{-} \mathrm{CR}^{\text {met }}(\mathrm{p}=0.078)$. For right and left side $\mathrm{CRC}^{\text {met }}$ tumors, right-sided tumors had a median OS of 21.4 months and had advantage over left-sided primary tumors with a median OS of 9.3 months $(\mathrm{p}=0.032)$. 
Pathologically lymphovascular invasion status, perineural invasion status, and MSI phenotype did not show any effect on OS or PFS. Similarly for clinical attributes; tumor's marker expression, location and initial region of metastasis did not differ regarding influence on PFS and OS with statistical significance.

Our one case has shown adenosquamous differentiation with BRAF V600E mutation. To the best of our knowledge, this is the first case in the literature. She was a 49-year-old female presenting colonic obstruction with peritoneal and liver metastases. The patient has died after five months of diagnosis because of ileus and post-renal acute renal failure.

\section{DISCUSSION}

BRAF mutation is now acknowledged as a consequential feature of colorectal cancer with impacts on prognosis and treatment. Nevertheless, its clinical utilization is still under debate probably owing to the fact that it embraces a heterogeneous group of tumors with also geographical diversity. In addition to that, as is known from other tumors, separate mutations on BRAF protein have different attributes. ${ }^{7,8,10}$ The aim of our study is to delineate the specific characteristics of nonV600E BRAF mutations on colorectal cancer and also describe characteristic features of V600E BRAF mutated colorectal cancer in Turkish population.

In our study, male to female ratio was found to be 1.15 for overall ${ }^{\mathrm{mt}} \mathrm{BRAF}$ tumors, for ${ }^{\mathrm{V} 600 \mathrm{E}} \mathrm{BRAF}$ it was 1.4. In Western populations BRAF mutation is known to be more frequent in females, although there are studies from Eastern populations showing male or no predominance. ${ }^{23-26}$ Median age of our patients was 59.5 years, compatible with literature. For the location of primary tumor, ${ }^{\mathrm{V}}{ }^{\mathrm{C}} \mathrm{CRC}$ tumors were more frequently located on proximal colon (60\%), contrastingly ${ }^{\mathrm{n}}{ }^{\mathrm{V} 600 \mathrm{E}} \mathrm{CRC}$ tumors significantly had predilection for distal colon (\%86.6) consistent with other studies regarding nonV600E tumors. ${ }^{9}$ Lymphovascular and perineural invasion did not differ between ${ }^{\mathrm{nonV}}{ }^{\mathrm{V} 600 \mathrm{E}} \mathrm{CRC}$ and ${ }^{\mathrm{V}}{ }^{600 \mathrm{E}} \mathrm{CRC}$ groups. Due to the retrospective nature of our study, our evaluation of the epigenetic attributes had to be limited. Our data indicate that MSI-H phenotype is also frequent in ${ }^{\mathrm{nonV}}{ }^{600 \mathrm{E}} \mathrm{CRC}$ tumors.
Adenosquamous differentiation is a rare attribute comprising $0.5-2 \%$ of colorectal cancer. ${ }^{27}$ It is more frequently seen in older age, male gender, and Caucasian population with a more advanced stage and undifferentiated histology, thus shorter OS. Correspondingly, our case is a 66 year-old male presenting with a rectal tumor and liver metastasic, and had a shorter OS of 5 months. To the best of our knowledge, this is the first case in literature reporting adenosquamous histology in a ${ }^{\mathrm{V} 600 \mathrm{E}} \mathrm{BRAF}$ mutant colorectal cancer.

In conclusion, for Turkish population, epidemiologic, pathologic and clinical characteristics of V600E mutation in colorectal cancer is consistent with literature with being seen more frequently in elderly and patients, proximal predominance, increased lymphovascular and perineural invasion, frequent MSI-H phenotype, and decreased overall survival.

There are only scarce data in literature regarding ${ }^{\mathrm{nonV} V 000} \mathrm{CRC}$. First study on this matter reviewed 10 metastatic colorectal cancer cases with BRAF codons 594 and 596 mutations. The cases were found to be rectal and left colon predominant, with nonmucinous histology and lower tumor-grade, having microsatellite stability and a longer OS compared to V600E mutant tumors. ${ }^{28}$ Another recently published study evaluated a retrospective cohort of 208

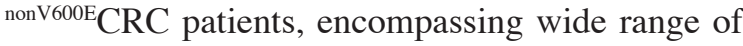
mutations, yielded similar results: distal predominance, microsatellite stability and longer OS with addition of male predominance and lesser propensity of peritoneal metastasis. ${ }^{9}$ Herein this study we have evaluated 11 cases of BRAF nonV600E mutant colorectal cancer, five of them being 597th codon mutated, remaining cases were mutated on 600th Codon. Considering all nonV600E BRAF mutations, our findings are partially compatible with previous studies as having less aggressive pathologic features, more propensity to be left sided and having a longer OS than V600E mutant tumors. Conversely nonv600E $C R C$ cases did not differ from ${ }^{\mathrm{V} 600 \mathrm{E}} \mathrm{CRC}$ in terms of patient age and gender. 597th codon mutations are not distinct from other mutations in any way with statistically significance. 
Uttermost pronounced limitation of our study is the low number of patients probably caused from the reported low prevalence of these mutations in colorectal cancer patients. Another further limitation is the retrospective nature of our study. There are also some missing data that was mentioned above resulted by unperformed further pathological evaluations deemed non-essential in clinical circumstances previously.

Much is known regarding V600E mutation in colorectal cancer (CRC): it is more frequently in female and elderly population, tumors tend to be proximal, histopathology is significant with lymphocytic infiltration and mucinous changes. Its genetic profile is high microsatellite instability (MSI-H) and increased $\mathrm{CpG}$ island methylation (CIMP-H) phenotype. Less is known for non-V600E mutation in CRC. Hereby in this detailed evaluation of non-V600E cases, we aimed to delineated this rare mutation in CRC. It was frequent in female and elderly, had predilection for distal colon, showed similar histopathologic attributes albeit with a better prognosis. Non-V600E BRAF mutation tends to carry a better prognostic significance and can be managed less aggressively.

Colorectal cancer is an important cause of cancer related mortality and morbidity. With our understanding of the footsteps of carcinogenesis, we have come a long way in its treatment. Nevertheless, ongoing discoveries of new molecular subtypes of colorectal cancer show that we have a long road ahead. Specifically, better knowledge of the mutations related to BRAF protein with higher patient numbers, longer follow-up periods and a more detailed genetic and pathologic analysis, has the potential to help us formulate new perspectives on treatment of colorectal cancer.

\section{REFERENCES}

1 Siegel RL, Miller KD, Jemal A. Cancer statistics, 2016. CA Cancer J Clin 66: 7-30, 2016.

2 Wan PT, Garnett MJ, Roe SM, et al. Mechanism of activation of the RAF-ERK signaling pathway by oncogenic mutations of B-RAF. Cell 116: 855-867, 2004.

3 Rowland A, Dias MM, Wiese MD, et al. Meta-analysis of BRAF mutation as a predictive biomarker of benefit from antiEGFR monoclonal antibody therapy for RAS wild-type metastatic colorectal cancer. Br J Cancer 112: 1888-1894, 2015.
4 Pietrantonio F, Petrelli F, Coinu A, et al. Predictive role of BRAF mutations in patients with advanced colorectal cancer receiving cetuximab and panitumumab: a meta-analysis. Eur J Cancer 51: 587-594, 2015.

5 Nelson DS, Quispel W, Badalian-Very G, et al. Somatic activating ARAF mutations in Langerhans cell histiocytosis. Blood 123: 3152-3155, 2014.

6 Siroy AE, Boland GM, Milton DR, et al. Beyond BRAFV600: Clinical Mutation Panel Testing by Next-Generation Sequencing in Advanced Melanoma. J Invest Dermatol 135: 508-515, 2015.

7 Menzies AM, Haydu LE, Visintin L, et al. Distinguishing clinicopathologic features of patients with V600E and V600K $<$ em>BRAF</em>-mutant metastatic melanoma. Clin Cancer Res 18: 3242-3249, 2012.

8 Marchetti A, Felicioni L, Malatesta S, et al. Clinical features and outcome of patients with non-small-cell lung cancer harboring BRAF mutations. J Clin Oncol 29: 3574-3579, 2011.

9 Jones JC, Renfro LA, Al-Shamsi HO, et al. Non-V600BRAF Mutations Define a Clinically Distinct Molecular Subtype of Metastatic Colorectal Cancer. J Clin Oncol 35: 2624-2630, 2017.

10 Shinozaki E, Yoshino T, Yamazaki K, et al. Clinical significance of BRAF non-V600E mutations on the therapeutic effects of anti-EGFR monoclonal antibody treatment in patients with pretreated metastatic colorectal cancer: the Biomarker Research for anti-EGFR monoclonal Antibodies by Comprehensive Cancer genomics (BREAC) study. Br J Cancer 117: 1450-1458, 2017.

11 Guinney J, Dienstmann R, Wang X, et al.The consensus molecular subtypes of colorectal cancer. Nat Med 21: 13501356, 2015.

12 Van Cutsem E, Dekervel J. Not all BRAF-mutant metastatic colorectal cancers are identical: distinct clinical consequences of (non-V600) BRAF Mutations. J Clin Oncol 35: 25982599, 2017.

13 Sanz-Garcia E, Argiles G, Elez E, Tabernero J. BRAF mutant colorectal cancer: prognosis, treatment, and new perspectives. Annal Oncol 28: 2648-2657, 2017.

14 Loupakis F, Cremolini C, Salvatore L, et al. FOLFOXIRI plus bevacizumab as first-line treatment in BRAF mutant metastatic colorectal cancer. European journal of cancer (Oxford, England : 1990) 50: 57-63, 2014.

15 Sanz-Garcia E, Argiles G, Elez E, Tabernero J. BRAF mutant colorectal cancer: prognosis, treatment, and new perspectives. Ann Oncol 28: 2648-2657, 2017.

16 Torre LA, Bray F, Siegel RL, et al. Global cancer statistics, 2012. CA Cancer J Clin 65: 87-108, 2015.

17 Favoriti P, Carbone G, Greco M, et al. Worldwide burden of colorectal cancer: a review. Upd Surg 68: 7-11, 2016. 
18 Phua LC, Ng HW, Yeo AH, et al. Prevalence of KRAS, BRAF, PI3K and EGFR mutations among Asian patients with metastatic colorectal cancer. Oncol Lett 10: 2519-2526, 2015.

19 Zhang J, Zheng J, Yang Y, et al. Molecular spectrum of KRAS, NRAS, BRAF and PIK3CA mutations in Chinese colorectal cancer patients: analysis of 1,110 cases. Sci Rep 5: 18678, 2015.

20 Siraj AK, Prabhakaran S, Bavi P, et al. Prevalence of Lynch syndrome in a Middle Eastern population with colorectal cancer. Cancer 121: 1762-1771, 2015.

21 Ozen F, Ozdemir S, Zemheri E, et al. The proto-oncogene KRAS and BRAF profiles and some clinical characteristics in colorectal cancer in the Turkish population. Genet Test Mol Biomarkers 17: 135-139, 2013.

22 Gorukmez O, Yakut T, Gorukmez O, et al. Distribution of KRAS and BRAF mutations in metastatic colorectal cancers in Turkish Patients. Asian Pac J Cancer Prev 17: 1175-1179, 2016.

23 Scartozzi M, Giampieri R, Aprile G, et al. The distinctive molecular, pathological and clinical characteristics of BRAF-mutant colorectal tumors. Expert Rev Mol Diagn 15: 979-987, 2015.

24 Vilkin A, Niv Y, Nagasaka T, et al. Microsatellite instability, MLH1 promoter methylation, and BRAF mutation analysis in sporadic colorectal cancers of different ethnic groups in Israel. Cancer 115: 760-769, 2009.

25 Saridaki Z, Tzardi M, Sfakianaki M, et al. BRAFV600E mutation analysis in patients with metastatic colorectal cancer $(\mathrm{mCRC})$ in daily clinical practice: correlations with clinical characteristics, and its impact on patients' outcome. PloS one 8: e84604, 2013.
26 Beg S, Siraj AK, Prabhakaran S, et al. Molecular markers and pathway analysis of colorectal carcinoma in the Middle East. Cancer 121: 3799-3808, 2015.

27 Masoomi H, Ziogas A, Lin BS, et al. Population-based evaluation of adenosquamous carcinoma of the colon and rectum. Dis Colon Rectum 55: 509-514, 2012.

28 Cremolini C, Di Bartolomeo M, Amatu A, et al. BRAF codons 594 and 596 mutations identify a new molecular subtype of metastatic colorectal cancer at favorable prognosis. Ann Oncol 26: 2092-2097, 2015

\section{Correspondence:}

\section{Dr. Cem SIMSEK}

Hacettepe Universitesi Tip Fakultesi

Gastroenteroloji Anabilim Dali

06100 Sihhiye, NKARA / TURKEY

Tel: (+90-534) 2964684

e-mail: cemgsimsek@gmail.com

\section{ORCIDs:}

Cem Simsek

Ece Esin

Sahin Lacin

Suayib Yalcin 